\title{
AOR
}

Selected Papers of \#AoIR2020:

The $21^{\text {st }}$ Annual Conference of the

Association of Internet Researchers

Virtual Event / 27-31 October 2020

\section{WHAT DO YOU WEIGH? POPULAR FEMINISM AND BODY POSITIVITY AS MEDIATED DISEMBODIMENT}

\author{
Hannah Taylor \\ Clemson University \\ Colten Meisner \\ Cornell University
}

On 16 March 2018, actress and activist Jameela Jamil posted a photo to Instagram, obscuring her body with textual identifiers like "great friends" and "I laugh every day." This post marked the launch of the Instagram account @i_weigh. This page, founded and maintained by Jamil, posts submissions from Instagram users answering the call to "weigh" themselves beyond the corporeal. This movement, now millions in reach, signals a departure from the conventions of body positivity.

Rather than discovering empowerment through the body, @i_weigh encourages its participants to publicly privilege external relationships, social identities, and economic opportunities in an effort to look past the body. Drawing on a visual discourse analysis of 300 Instagram posts from the @i_weigh account, this study suggests that body positivity movements may be increasingly disembodied in a self-representational era hallmarked by popular feminism.

\section{Body Positivity as an Expression of Popular Feminism}

Popular feminism (Banet-Weiser, 2018) situates feminist politics in a neoliberal culture by directing the means of empowerment for women and girls to a visibility economy. Body positivity movements are a visible expression of popular feminism in that they require the body to be put on display in a digital space, simultaneously requiring visibility while also enabling body image vulnerabilities. Body positivity initially rose to prominence as a component of the radical and fringe ideologies that included public anti-discrimination protests and anti-capitalist advocacy against the diet industry (Mull, 2018). The original intent of body positivity was to subvert. That is, to have a body that is marginalized and reviled in a world that affronts a person with shame and guilt-laden cultural values about their body_and accept it anyway_is inherently subversive. 
However, contemporary body positivity movements both commodify the body through visibility and place responsibility back on the individual. It asks women, in particular, to change their own self-image, directing critical attention away from the structures that created the need for empowerment in the first place. The move to a neoliberal sensibility of individual responsibility, and emphasis on capitalism, places body positivity discourses squarely in the domain of popular feminism. Although body positivity has been primarily steeped in concerns of appearance, @i_weigh takes a different approach, seeking instead to overlook the body while inevitably underscoring its importance.

\section{Method}

Data from this project come from self-representational, user-submitted posts on the Instagram account @i_weigh. The page instructs contributors to submit a photo where textual labels (i.e., non-physical "weights") are superimposed over their bodies. After removing duplicate posts and advertisements, researchers collected the first 300 posts from the account, dated 16 March 2018 to 19 April 2018. Images and textual identifiers were analyzed using an approach informed by visual discourse analysis, wherein visual representations index larger constructions of power and inequality (Rose, 2007).

\section{Findings: Liminality, Contradictory Self-Presentation, and Mediated Disembodiment}

The women, and few men, who contributed to @i_weigh exemplify identification in the age of popular feminism wherein the individual subject is positioned as responsible for their own empowerment and asked to define themselves publicly in this way. Our findings suggest that @i_weigh discursively constructs a mediated disembodiment for participants, characterized by its liminal visual representation and categorization of the self through narratives of resilience and strength, claiming marginalized identities, and extra-self connection.

The visual analysis of the posts revealed a disconnect between the body and the identities that the posters "weighed" heavily. Most posts featured a person both literally and metaphorically covered, a deviation from the more normalized conceptions of body positivity which insist on visibility. Though faces were captured, they were frequently obscured by the "weight" of the contributor. The text is superimposed over the image and often in a bright color, drawing the eye of the viewer immediately. The text is visually more important and central than the body. Most of the posts do not depict the body doing anything. It is a fixed subject, operating almost as a lifeless backdrop to the words on the screen. The image in black and white reinforces the static nature of the body-dull and lifeless, hidden like a ghostly presence behind the words. The separation of the body from the self-identification of the participants demonstrates a liminality present in body positive discourses.

Most experiences of liminality (Härkönen \& Räsänen, 2008; Little, Jordens, Montgomery, \& Philipson, 1998; van Gennep 1960) are focused on not being defined at all by the body, removing any value placed on an individual's physical experiences. Although this is not problematic at face value, it leads to a self-conception that inhibits 
embodiment. Within this discourse, the inner self is seen as a valid subject, while the physical body is deemed other and stigmatized. The familiar problem with viewing the body in a liminal state is that it denies that body work, or that anyway a body functions, could be integral to a person's experience and identity. Particularly for people with marginalized identities, forcing a racialized, queered, or fat body in a liminal sphere can result in internalized hatred.

The words chosen by the participants emerged in three primary categories: narratives of resilience and strength, claiming marginalized identities, and extra-self connection. The first theme focused on contributors disclosing mental and physical illness as part of their self-presentation, as well as repeated mention of recovery and survival from abuse, addiction, and trauma. The posters also identified with other marginalized communities, including but not limited to racial, queer, and activist communities. They often reference their external connections, including family, friends, romantic partnerships, and fandoms. This final note creates a contradictory dialectic of self-presentation, as they are valuing communal identities that are technically outside of the self.

The forced presentation of the mind as separated from the body is a mediated disembodiment facilitated by the platform - a guiding of the individual to consider their body as deviant and valueless, their mind as rational and "true," all the while preventing the disruption of ideal beauty norms. This mediated disembodiment inhibits participants from experience embodied body positivity, because the body is marked as inferior within this definition of self. The interaction of liminality and mediated disembodiment is reflective of self-representation in an age of popular feminism, placing the responsibility back on women for their own empowerment and production of selfhood, while ignoring the sociocultural frameworks that create a need for empowerment in the first place.

\section{References}

Banet-Weiser, S. (2018). Empowered: Popular feminism and popular misogyny. Durham, NC: Duke University Press.

Härkönen, J., \& Räsänen, P. (2008). Obesity, unemployment and earnings. Work Life Research, 6(1), 3-16.

Little, M., Jordens, P., Montgomery, K., \& Philipson, B. (1998). Liminality: A major category of experience of cancer illness. Social Science \& Medicine, 47(10), $1485-1494$.

Mull, A. (2018, June 5). Body positivity is a scam. Vox. Retrieved from https://www.vox.com/2018/6/5/17236212/body-positivity-scam-dove-campaignads

Rose, G. (2007). Discourse analysis I: Text, intertextuality and context. In Visual methodologies: An introduction to the interpretation of visual materials (2nd ed., pp. 141-171). Thousand Oaks, CA: Sage. 
van Gennep, A. (1960/1909). Rites of passage. Chicago: University of Chicago Press. 